\title{
纳米结构二硫化钼的制备及其应用
}

\author{
翟英娇 ${ }^{1}$ ，李金华 ${ }^{1}$ ，楚学影 ${ }^{1}$ ，徐铭泽 ${ }^{1}$ ，李 雪 ${ }^{1,2}$ ，方 铉 $^{1,3}$ ，魏志鹏 ${ }^{3}$ ，王晓华 ${ }^{3}$
}

(1. 长春理工大学 国际纳米光子学与生物光子学联合研究中心, 长春 $130022 ; 2$. 吉林农业大学信息技术学院, 长春 130118; 3. 高功率半导体激光国家重点实验室, 长春 130022)

摘 要: 二硫化钼是一种典型的过渡金属二元化合物, 以其独特的化学、物理性能而备受关注。本文综述了纳米二 硫化钼常见的多种形貌结构，包括富勒烯状、球状、花状、线、片、棒、管状等; 概述了其常用的制备方法，包括： 化学气相沉积法、高温硫化法、剥离法、电化学沉积法、水热及溶剂热法等; 总结了纳米结构二硫化钼在润滑、催 化、光电器件等领域的研究进展, 最后展望了二硫化钼材料的研究前景。

关 键 词: 二硫化钼; 形貌结构; 材料制备; 光电器件; 综述

中图分类号: O469 文献标识码: A

\section{Preparation and Application of Molybdenum Disulfide Nanostructures}

\author{
ZHAI Ying-Jiao ${ }^{1}$, LI Jin-Hua ${ }^{1}$, CHU Xue-Ying ${ }^{1}$, XU Ming-Ze ${ }^{1}$, LI Xue ${ }^{1,2}$, \\ FANG Xuan ${ }^{1,3}$, WEI Zhi-Peng ${ }^{3}$, WANG Xiao-Hua ${ }^{3}$
}

(1. School of Science, International Joint Research Center for Nanophotonics and Biophotonics, Changchun University of Science and Technology, Changchun 130022, China; 2. College of Information Technology, Jilin Agriculture University, Changchun 130118, China; 3. State Key Laboratory of High Power Semiconductor Laser, Changchun University of Science and Technology, Changchun 130022, China)

\begin{abstract}
Molybdenum disulfide $\left(\mathrm{MoS}_{2}\right)$, a new binary transition-metal compound, has attracted much attention due to its unique physical and chemical properties. $\mathrm{MoS}_{2}$ with different morphology structures were firstly reviewed, including inorganic fullerene-like, sphere-like, flower-like, wire, plate, rod, tube and so on. And then, the methods used to prepare $\mathrm{MoS}_{2}$ nanomaterials were discussed, including chemical vapor deposition method, sulfurization process, exfoliation, electrodeposition, hydrothermal method, and solvothermal method. The applications of $\mathrm{MoS}_{2}$ nanostructures in various fields were also summaried, such as lubrication, catalytic and photoelectric devices. Finally, the outlook for the research of this molybdenum disulfide nanomaterial was proposed.
\end{abstract}

Key words: molybdenum disulfide; morphology structure; material preparation; photoelectric device; review

石墨烯具有优异的光电性能，吸引了广泛关注， 但该材料为零带隙材料, 缺少能带隙, 限制了其在 光电器件等方面的应用。过渡金属二元化合物 $\left(\mathrm{MX}_{2}\right)$ 不仅具有与石墨烯相似的层状结构, 并且在润滑、 催化、光电器件等方面拥有独特的性能, 成为了国 内外研究热点。二硫化钼 $\left(\mathrm{MoS}_{2}\right)$ 作为一种典型的过
渡金属二元化合物，具有类石墨烯结构，层内 Mo 与 $\mathrm{S}$ 原子之间构成共价键，结构稳定，单层 $\mathrm{MoS}_{2}$ 的 厚度为 $0.65 \mathrm{~nm}$ 。类石墨烯 $\mathrm{MoS}_{2}$ 具有一定的带隙能 $(1.2 \sim 1.9 \mathrm{eV})$ 。此外, Mo 和 $\mathrm{S}$ 为天然矿物, 储量丰富, 价格低廉, 增强了 $\mathrm{MoS}_{2}$ 在光电器件方面应用的可 行性 ${ }^{[1]}$ 。

收稿日期: 2015-03-11; 收到修改稿日期：2015-04-24

基金项目: 国家自然科学基金(61204065，61205193，61307045，61404009); 吉林省科技发展计划(20140520107JH, 20140204025GX) National Natural Science Foundation of China (61204065, 61205193, 61307045, 61404009); Developing Project of Science and Technology of Jilin Province (20140520107JH, 20140204025GX)

作者简介: 翟英娇(1988-), 女，博士研究生. E-mail: zhaiyingjiao0613@sina.com

通讯作者: 李金华, 教授.E-mail: jhli_cust@163.com 
虽然 $\mathrm{MoS}_{2}$ 具有巨大的应用潜力，但存在着制 备困难等问题, 研究人员需要寻找更为有效的 $\mathrm{MoS}_{2}$ 的制备方法, 弥补工艺制备方法中的不足, 提 高制备样品的质量, 从而更好地应用于工业生产 中。本文将对 $\mathrm{MoS}_{2}$ 的结构特性、形貌、制备方法 及应用等方面展开介绍。

\section{1 二硫化钼的结构及特性}

$\mathrm{MoS}_{2}$ 为黑色固体粉末, 具有金属光泽, 其化学 和热稳定性良好, 可广泛应用于固体润滑剂和催化 剂等方面。 $\mathrm{MoS}_{2}$ 价格低廉, 不溶于水, 但溶于王水、 浓硫酸 ${ }^{[2]}$ 。单层 $\mathrm{MoS}_{2}$ 的结构与石墨烯类似, 层内 Mo 原子与 $\mathrm{S}$ 原子之间形成共价键, 且结构稳定, 晶 格参数为 $a=b=0.3160 \mathrm{~nm}, c=1.2294 \mathrm{~nm}, w=0.2975 \mathrm{~nm}$, $\mathrm{z}=0.1586 \mathrm{~nm}$ 。

与层状石墨烯结构相比, 类石墨烯 $\mathrm{MoS}_{2}$ 具有 特殊的能带结构, 其布里渊区的能带为一个平面, 该中心与其平面上的任一点连线均构成一个波数矢 量 $(\mathrm{k}$ 矢量), 分别对应一个相应的能级 $E(\mathrm{k})$, 故类石 墨烯 $\mathrm{MoS}_{2}$ 的能带展开图为布里渊区平面沿着高对 称点的展开 ${ }^{[3]}$ 。类石墨烯 $\mathrm{MoS}_{2}$ 具有一定的带隙能 $(1.29 \sim 1.90 \mathrm{eV})$, 其体材料为间接带隙半导体材料, 禁带宽度为 $1.29 \mathrm{eV}$, 随着尺度的变小, 单层 $\mathrm{MoS}_{2}$ 转变为直接带隙材料, 禁带宽度为 $1.90 \mathrm{eV}$, 故由于 类石墨烯 $\mathrm{MoS}_{2}$ 的能带隙可调, 在光电器件方面具 有可观的发展前景。

六方晶系结构的 $\mathrm{MoS}_{2}$ 具有各向异性的类石墨 烯层状结构, 金属 Mo 原子层夹在两个 $\mathrm{S}$ 原子层中 间, 形成典型的 “三明治” 结构。Mo-S 键较短, 而 $\mathrm{S}-\mathrm{S}$ 键长较长, 在较强的共价键作用下, 形成 S-Mo-S 键且在(001)的晶面方向沿着六方对称的结 晶学原理进行堆叠。在层状 $\mathrm{MoS}_{2}$ 中, $\mathrm{Mo}$ 原子为六 配位, 形成 $1 \mathrm{~T} 、 2 \mathrm{H} 、 3 \mathrm{R}$ 型三种晶体结构, 如图 1 所示。1 $1 \mathrm{~T}-\mathrm{MoS}_{2}$ 为亚稳性, 晶型结构具有金属性, Mo 原子为八面体配位, 晶胞由 1 个 $\mathrm{S}-\mathrm{Mo}-\mathrm{S}$ 单分子层组
$1 \mathrm{~T}$

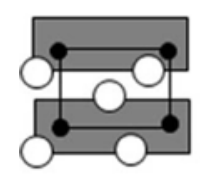

$2 \mathrm{H}$

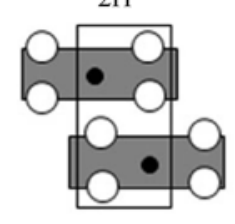

$3 \mathrm{R}$

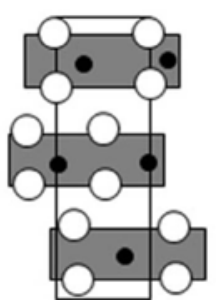

图 1 六方晶系 $\mathrm{MoS}_{2}$ 三种晶型的结构模型

Fig. 1 Three kinds of hexagonal $\mathrm{MoS}_{2}$ crystal structure model
成。 $2 \mathrm{H}-\mathrm{MoS}_{2}$ 为稳定相, 晶型结构具有半导体性, Mo 原子为三角棱柱配位, 晶胞由 2 个 S-Mo-S 单分 子层组成, 常温下存在典型的层状结构。3R-MoS 也为亚稳性, Mo 原子为三角棱柱配位, 晶胞由 3 个 $\mathrm{S}-\mathrm{Mo}-\mathrm{S}$ 单分子层组成 ${ }^{[4]}$ 。

\section{2 二硫化钼的形貌结构}

$\mathrm{MoS}_{2}$ 作为一种典型的过渡金属硫化物，早期 由于其良好的润滑性而备受关注，后来随着纳米科 技的兴起, $\mathrm{MoS}_{2}$ 也在纳米尺度范围的研究中开拓了 新的领域。1993 年 Tenne 等首次制备出富勒烯状 $\mathrm{MoS}_{2}$ 纳米粒子, 因其特有性质引起广大研究人员 的关注。

为了更好地研究不同形貌 $\mathrm{MoS}_{2}$ 的相关特性, 国内外研究人员通过改变 $\mathrm{pH}$ 、添加剂、反应温度和 反应时间等实验条件制备了球、花、线、片、棒和 管状等结构的 $\mathrm{MoS}_{2}$ 纳米材料。Tan 等 ${ }^{[5]}$ 利用 $\mathrm{H}_{2} \mathrm{C}_{2} \mathrm{O}_{4}$ 调节 $\mathrm{pH}$ ，通过水热法成功制备了 $\mathrm{MoS}_{2}$ 微米球和纳 米球的结合体(图 2(a))。Zhu 等 ${ }^{[6]}$ 利用模板通过化学 气相沉积法(CVD)成功获得了玫瑰花瓣状分层纳米 结构 $\mathrm{MoS}_{2}$, 在析氢反应中表现出优异的结构特性 (图 2(b))。Chen 等 ${ }^{[7]}$ 通过低温硫化作用成功制备了 面向垂直的 $\mathrm{MoO}_{3}-\mathrm{MoS}_{2}$ 核-壳纳米线, $\mathrm{MoO}_{3}$ 核层材 料可更好地进行电荷传输, 而 $\mathrm{MoS}_{2}$ 壳层材料具有 优异的催化活性, 且在强酸中抗腐蚀能力较强(图 $2(\mathrm{c}))$ 。 $\mathrm{Wu}$ 等 ${ }^{[8]}$ 以 $\mathrm{MoO}_{3}$ 和单质 $\mathrm{S}$ 为原料, 在氩气氛 围中球磨 $24 \mathrm{~h}$, 然后在氩气保护的管式炉中 $900^{\circ} \mathrm{C}$ 保持 $1 \mathrm{~h}$, 利用固态组装法获得六角的 $\mathrm{MoS}_{2}$ 纳米片, 其尺寸约为 $500 \mathrm{~nm}$, 且沿 $c$ 轴方向堆叠生长。 Lin 等 ${ }^{[9]}$ 以硅铇酸为添加剂，在聚四氟乙烯高压釜中加 热至 $220^{\circ} \mathrm{C}$ 反应 $24 \mathrm{~h}$, 制备出直径约为 20 50 nm, 长 度达约 400 500 nm 的 $\mathrm{MoS}_{2}$ 纳米棒。Deepak 等 ${ }^{[10]}$

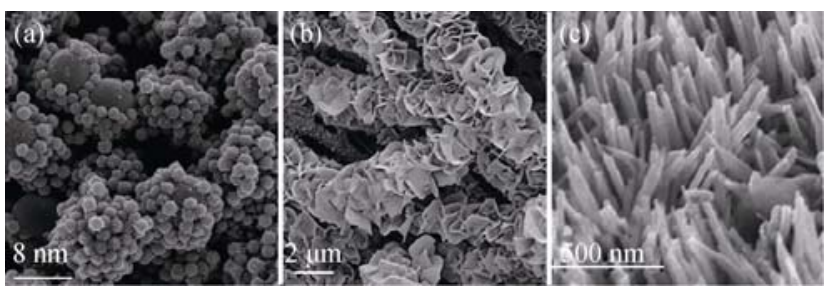

图 2 (a) $\mathrm{MoS}_{2}$ 空心微米球和纳米球的结合体的 SEM 照片 ${ }^{[5]}$; (b) 玫瑰花瓣状 $\mathrm{MoS}_{2}$ 的 SEM 照片 ${ }^{[6]}$; (c) $\mathrm{MoO}_{3}-\mathrm{MoS}_{2}$ 核-壳纳 米线的 SEM 照片 ${ }^{[7]}$

Fig. 2 (a) SEM images of a hollow $\mathrm{MoS}_{2}$ micro@nanospheres ${ }^{[5]}$; (b) Rose-petal-shaped $\mathrm{MoS}_{2}$ hierarchical nanostructures ${ }^{[6]}$; (c) Core-shell $\mathrm{MoO}_{3}-\mathrm{MoS}_{2}$ nanowires ${ }^{[7]}$ 
在 $1000^{\circ} \mathrm{C}$ 的管式炉中通入 $\mathrm{N}_{2}, \mathrm{MoO}_{3}$ 和 $\mathrm{CSN}_{2} \mathrm{H}_{4}$ 通过 化学气相沉积法充分反应, 获得高内表面积的 $\mathrm{MoS}_{2}$ 纳米管，纳米管直径为 200 800 nm。

\section{3 二硫化钼的制备}

$\mathrm{MoS}_{2}$ 纳米材料的制备方法根据前驱体的不同 可以分为天然法和人工合成法。天然法制备 $\mathrm{MoS}_{2}$ 是采用浮选法、浸出剂、烘干提纯和机械研磨等方 法获得纳米级 $\mathrm{MoS}_{2}$ 。此方法虽然可以大批量制备 $\mathrm{MoS}_{2}$, 但需要大量天然精矿, 生产成本高, 制约着 $\mathrm{MoS}_{2}$ 的应用前景, 所以研究人员力求研究出简单、 低廉、产率高的制备方法。

目前 $\mathrm{MoS}_{2}$ 的制备方法层出不穷, 大致可分为 化学合成法和物理合成法。化学合成法可以更好地 控制纳米材料的结构、形貌、表面性能等, 主要包 括气相法、液相法与固相法等 ${ }^{[11-14]}$, 其中气相法主 要有化学气相沉积法、高温硫化法等; 液相法则包 括水热法、溶剂法、模板法、溶胶一凝胶等。常见 的物理合成方法则有物理气相沉积法、机械研磨 法、剥离法等。下面将对常见的制备方法进行简单 的介绍。

\section{1 化学气相沉积法}

化学气相沉积法, 即固态硫源和钼源在高温情 况下升华为气态的过程, 通过改变保护气体的比例 来控制纳米 $\mathrm{MoS}_{2}$ 的结构 ${ }^{[15]}$ 。Shi 等 ${ }^{[16]}$ 利用低压化学 气相沉积法(LPCVD)在商购 $\mathrm{Au}$ 箔片上成功制备了 单层 $\mathrm{MoS}_{2}$ (图 3(a)), 通过改变生长温度或者祄底位 置使三角型单层 $\mathrm{MoS}_{2}$ 薄片尺寸由纳米级 $(200 \mathrm{~nm}$, 图 3(b))变为微米级。Zhan 等 ${ }^{[17]}$ 通过化学气相沉积 法在 $\mathrm{SiO}_{2}$ 祄底上大面积生长 $\mathrm{MoS}_{2}$ 原子层, 成功获 得了厚度为 $1 \sim 5 \mathrm{~nm}$ 的 $\mathrm{MoS}_{2}$ 原子层。

\section{2 高温硫化法}

高温硫化法, 即在高温环境、还原性气体保护 下, 将钼源 $\left(\mathrm{MoO}_{3} 、 \mathrm{MoCl}_{3}\right.$ 等)中的六价钼还原到四

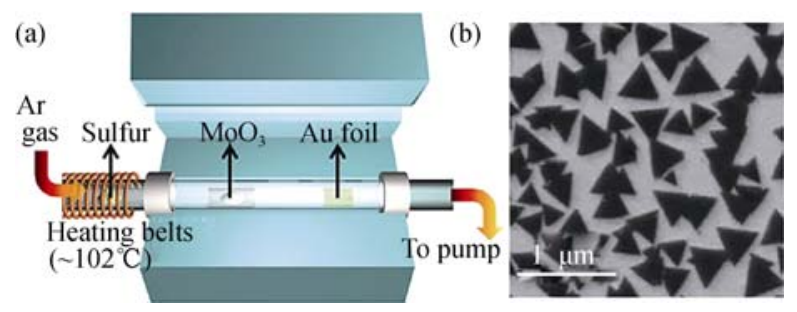

图 3 (a) LPCVD 设备实验装置图和(b) 生长温度为 $530^{\circ} \mathrm{C}$ 时 三角型单层 $\mathrm{MoS}_{2}$ 薄片的 SEM 照片 ${ }^{[16]}$

Fig. 3 (a) Experimental setup of the LPCVD system and (b) SEM image of $\mathrm{MoS}_{2}$ triangular flakes grown under temperature of $530^{\circ} \mathrm{C}^{[16]}$
价钼, 然后在硫源 $\left(\mathrm{H}_{2} \mathrm{~S}\right.$ 、气态 $\mathrm{S}$ 单质)的硫化作用下, 制得纳米 $\mathrm{MoS}_{2}{ }^{[18]}$ 。钿源为 $\mathrm{MoO}_{3}$, 硫源为 $\mathrm{H}_{2} \mathrm{~S}$ 时硫 化的反应机理为:

$$
\begin{gathered}
\mathrm{MoO}_{3}+2 \mathrm{H}_{2} \mathrm{~S}+\mathrm{H}_{2}=\mathrm{MoS}_{2}+3 \mathrm{H}_{2} \mathrm{O} \\
\mathrm{MoO}_{3}+3 \mathrm{H}_{2} \mathrm{~S}=\mathrm{MoS}_{2}+3 \mathrm{H}_{2} \mathrm{O}+\frac{1}{8} \mathrm{~S}_{8}
\end{gathered}
$$

钿源为 $\mathrm{MoO}_{3}$, 硫源为 $\mathrm{S}$ 单质时硫化的反应机理为:

$$
2 \mathrm{MoO}_{3}+\frac{7}{8} \mathrm{~S}_{8}=2 \mathrm{MoS}_{2}+3 \mathrm{SO}_{2}
$$

Kong 等 ${ }^{[19]}$ 在还原性气体( $\mathrm{Ar}$ 气)的保护下, 将 钼源在 $550^{\circ} \mathrm{C}$ 反应 $20 \mathrm{~min}$, 然后在硫源中 $220{ }^{\circ} \mathrm{C}$ 硫 化成垂直排列层状 $\mathrm{MoS}_{2}$ 薄膜，长度约 $10 \mathrm{~nm}$ (图 4)。 $\mathrm{Cai}$ 等 ${ }^{[20]}$ 将钼源 $\left(\mathrm{MoO}_{3}\right)$ 在空气环境下加热至 $750^{\circ} \mathrm{C}$ 并保持 $8 \mathrm{~h}$ 后, 自然冷却至室温形成白色或黄色纤 维状样品, 在单质硫的硫化作用下 $\left(350 \sim 1100^{\circ} \mathrm{C}\right.$, $4 \sim 10 \mathrm{~h}$ )制得六角形 $\mathrm{MoS}_{2}$ 微米片, 其中 $850 \mathrm{~K}$ 硫化 $8 \mathrm{~h}$ 制备的样品生长较为匀称。

\section{3 剥离法}

剥离法制备的纳米 $\mathrm{MoS}_{2}$ 结晶度好, 质量高, 无 杂质, 且操作简单, 适用于制备单个器件, 但也存 在一些不足, 如难以重复、不能批量生产等 ${ }^{[21]}$ 。Eda 等 ${ }^{[22]}$ 将反应物置于惰性气体 $(\mathrm{Ar}$ 气)环境下反应 $2 \mathrm{~d}$, 得到剥离插层物 $\mathrm{Li}_{x} \mathrm{MoS}_{2}$, 然后将样品清洗至中性 成功制得了层状 $\mathrm{MoS}_{2}$ (图 5(a)), $300^{\circ} \mathrm{C}$ 退火后剥离 材料由亚金属相转变为半导体的相。Liu 等 ${ }^{[23]}$ 通过 电化学剥离法成功制备了大面积的 $\mathrm{MoS}_{2}$ 纳米片, $\mathrm{MoS}_{2}$ 与 $\mathrm{Pt}$ 线间为直流偏压(图 5(b))。Yin 等 ${ }^{[24]}$ 采用 机械剥离法成功制备了单层 $\mathrm{MoS}_{2}$, 厚度约为 $0.8 \mathrm{~nm}$ (图 5(c))。Gopalakrishnan 等 ${ }^{[25]}$ 通过液相剥离法成 功制备了 $\mathrm{MoS}_{2}$ 量子点点缀的层状纳米结构 $\mathrm{MoS}_{2}$, $\mathrm{MoS}_{2}$ 量子点尺寸约为 $2 \mathrm{~nm}, \mathrm{MoS}_{2}$ 片横向尺寸约为 $1 \mu \mathrm{m}$ (图 5(d))。

\section{4 电化学沉积法}

电化学沉积法是可以自发进行的一种氧化还原

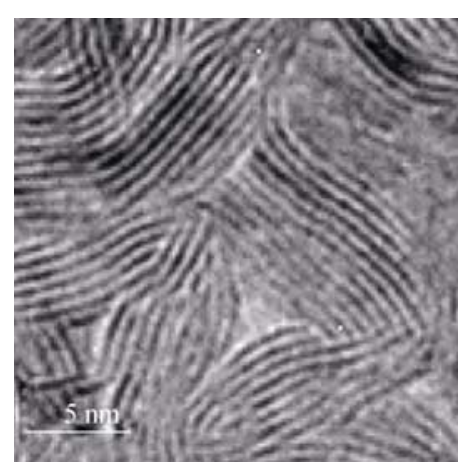

图 $4 \mathrm{MoS}_{2}$ 薄膜的 TEM 照片 ${ }^{[19]}$

Fig. 4 TEM image of $\mathrm{MoS}_{2}$ films ${ }^{[19]}$ 

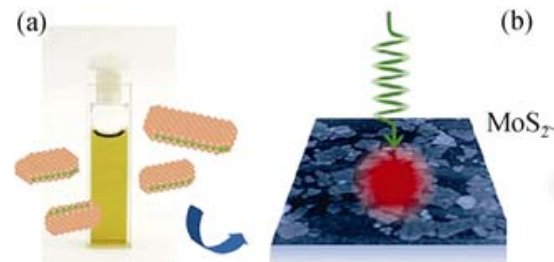

b) + DC Bias

(c)

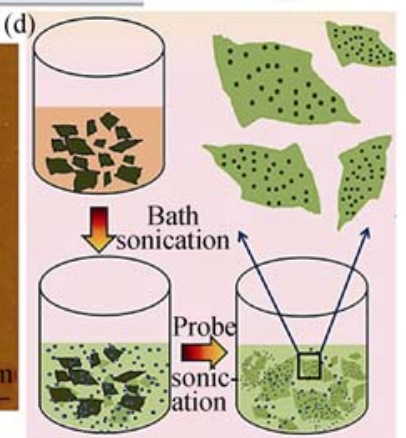

图 5 (a)化学剥离层状 $\mathrm{MoS}_{2}$ 设备实验装置图 ${ }^{[22]}$, (b)电化学剥离 块状 $\mathrm{MoS}_{2}$ 晶体的实验装置图 ${ }^{[23]}$, (c)单层 $\mathrm{MoS}_{2}$ 的 $\mathrm{AFM}$ 照片 ${ }^{[24]}$ 和(d) $\mathrm{MoS}_{2}$ 量子点点缀的 $\mathrm{MoS}_{2}$ 纳米片的实验装置图 ${ }^{[25]}$

Fig. 5 (a) Experimental setup of the chemically exfoliated $\mathrm{MoS}_{2}$ system $^{[22]}$; (b) Schematic illustration of experimental setup for electrochemical exfoliation of bulk $\mathrm{MoS}_{2}$ crystal $^{[23]}$; (c) AFM image of single-layer $\mathrm{MoS}_{2}{ }^{[24]}$; (d) Schematic representation of the synthesis procedure to obtain $\mathrm{MoS}_{2}$ quantum dots interspersed in $\mathrm{MoS}_{2}$ nanosheets ${ }^{[25]}$

反应，即在适当装置和电解液间构成原电池，从而 在电极上发生化学反应 ${ }^{[26]}$ 。 $\mathrm{Li}$ 等 ${ }^{[27]}$ 在大小可调的高 定向热解石墨(HOPG)表面通过电沉积法成功制备 了 $\mathrm{MoO}_{2}$ 纳米线, 然后在 $800 \sim 900{ }^{\circ} \mathrm{C} 、 \mathrm{H}_{2} \mathrm{~S}$ 氛围下转 变为 $\mathrm{MoS}_{2}, \mathrm{MoS}_{2}$ 纳米带为 $2 \mathrm{H}$ 型晶体结构, 厚度为 2 30 nm(图 6(a))。Kibsgaard 等 ${ }^{[28]}$ 采用模板与电沉 积法结合成功合成了双重螺旋二十四面体 $\mathrm{MoS}_{2}$,
$\mathrm{MoS}_{2}$ 网状物厚度约为 $4 \mathrm{~nm}$ ，宽度约为 $3 \mathrm{~nm}$ ，孔间 的距离为 $7 \mathrm{~nm}$ (图 6(b))。

\section{5 水热和溶剂热法}

水热和溶剂热法是指在高压釜中加入钼酸 盐和硫化物, 以去离子水或者有机溶剂作为溶剂, 高温高压下经过一系列物理化学反应合成多种 形态的硫化钿粉体，制得的样品尺寸可调，且纯 度较高 ${ }^{[29-32]}$ 。 $\mathrm{Li}$ 等 ${ }^{[33]}$ 以 $\left(\mathrm{NH}_{4}\right)_{2} \mathrm{MoS}_{4}$ 、水合肼为原料, 以 $\mathrm{N}, \mathrm{N}-$ 二甲基甲酰胺(DMF)为溶剂，通过溶剂热法 合成了 $\mathrm{MoS}_{2}$ 粒子(图 7)。Tang 等 ${ }^{[34]}$ 在 $180^{\circ} \mathrm{C}$ 条件下 水热反应生成花状纳米结构 $\mathrm{MoS}_{2}$, 通过改变 $\mathrm{pH}$ 值、 表面活性剂的剂量研究其形貌变化。

\section{4 二硫化钼的应用}

19 世纪中期, 美国将具有较弱范德华力的层状 $\mathrm{MoS}_{2}$ 用于马车的轴承上, 从而达到降低摩擦力的 作用。随着科技的快速发展, $\mathrm{MoS}_{2}$ 纳米材料在诸多 方面有着重要的应用，如：润滑剂、催化剂、涂层材 料和电子探针等; 此外由于 $\mathrm{MoS}_{2}$ 为 $\mathrm{P}$ 型窄带隙半 导体纳米材料, 也广泛应用于太阳能电池、光电器 件等方面。

\section{1 摩擦学应用}

$2 \mathrm{H}-\mathrm{MoS}_{2}$ 为典型的层状结构, 其夹心层的上层 $\mathrm{S}$ 原子的弧对电子恰好延伸进上一夹心层的下层 $\mathrm{S}$ 原子群组成的带负电空穴区，反之亦然，故 $2 \mathrm{H}$ $\mathrm{MoS}_{2}$ 由于静电排斥作用而容易剪切，从而具有优 异的润滑作用。而 $1 \mathrm{~T}-\mathrm{MoS}_{2}$ 则为其夹心层的下层 $\mathrm{S}$ (a)

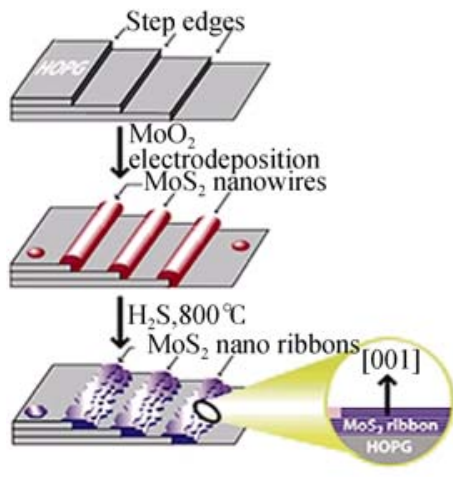

(b)

Dip-coat surfactant and Si precursor onto FTO slide
Surfactant and Si precursor DG silica template

DG silica template with $\mathrm{MoO}_{3-x}(\mathrm{OH})$

图 6 (a)电沉积/化学方法合成 $2 \mathrm{H}-\mathrm{MoS}_{2}$ 纳米带设备实验装置图 ${ }^{[27]}$ 和(b)双重螺旋二十四面体 $\mathrm{MoS}_{2}$ 的合成过程、结构模型 ${ }^{[28]}$

Fig. 6 (a) Electrochemical/chemical method for synthesizing $2 \mathrm{H}-\mathrm{MoS}_{2}$ nanoribbons $^{[27]}$ and (b) synthesis procedure and structural model for double-gyroid $\mathrm{MoS}_{2}{ }^{[28]}$ 
in DMF

solvothermal $200{ }^{\circ} \mathrm{C}$

$\left(\mathrm{NH}_{4}\right)_{2} \mathrm{MoS}_{4}$

Free $\mathrm{MoS}_{2}$

图 7 溶剂法合成 $\mathrm{MoS}_{2}$ 粒子的原理图 ${ }^{[33]}$

Fig. 7 Schematic solvothermal synthesis with $\mathrm{MoS}_{2}$ particles ${ }^{[33]}$

原子延伸进下一夹心层的上层 $\mathrm{S}$ 原子组成带正电空 穴区，同理，夹心层的下层 $\mathrm{S}$ 原子又延伸到上层 $\mathrm{S}$ 原子组成的带正电的空穴区中, 所以 $1 \mathrm{~T}-\mathrm{MoS}_{2}$ 由于 静电吸引作用, 增加抗剪切能力, 润滑性降低。 $\mathrm{MoS}_{2}$ 以其优异性质, 可被用作固体润滑剂、润滑 油、润滑脂的添加剂等。由于在高载荷、高温环境 中无法使用液体润滑, Rapoport 等 ${ }^{[35]}$ 成功制备了大 批量的富勒烯状 $\mathrm{MoS}_{2}$ 纳米粒子作为固体润滑剂, 并研究其作用机理。

\section{2 催化剂应用}

纳米 $\mathrm{MoS}_{2}$ 为六方结构, 晶体楞角较多, 比表面 积较大, 故在催化剂方面具有一定的应用前景 ${ }^{[36]}$ 。 $\mathrm{Li}$ 等 ${ }^{[37]}$ 采用两步水热法成功制备了新型 $\mathrm{MoS}_{2} @ \mathrm{SnO}_{2}$ 纳米花, 光照 $100 \mathrm{~min}$ 后对甲基蓝溶液的降解率高达 90.5\%(图 8(a))。Tan 等 ${ }^{[38]}$ 通过粘合剂成功合成了 $\mathrm{MoS}_{2}$ @ $\mathrm{ZnO}$ 纳米异质结, 其对甲基蓝溶液有明显的降解作 用, 光照 $100 \mathrm{~min}$ 后降解率高达 $92.7 \%$ (图 8(b))。

\section{3 光电器件方面应用}

$\mathrm{MoS}_{2}$ 作为直接带隙半导体, 具有独特的光电 特性, 在光电器件方面具有巨大的潜能 ${ }^{[39]}$ 。

(a) 场效应晶体管

$\mathrm{Kwak}$ 等 ${ }^{[40]}$ 在绝缘 $/ \mathrm{p}^{+}-\mathrm{Si}$ 祄底上成功构造了多层 $\mathrm{MoS}_{2}$ /石墨烯异质结器件, 如图 9(a)所示, 在\#1 和\#2
间形成石墨烯场效应晶体管, \#3 和\#4 之间形成 $\mathrm{MoS}_{2}$ 场效应晶体管, 沟道长度约为 10 20 $\mu \mathrm{m}$ 。LopezSanchez 等 ${ }^{[41]}$ 利用单层 $\mathrm{MoS}_{2}$ 成功制备了光敏晶体 管并应用于光电检测, 结果表明单层 $\mathrm{MoS}_{2}$ 光电晶 体管在 $561 \mathrm{~nm}$ 处光响应速度高达 $880 \mathrm{~A} / \mathrm{W}$ (图 9(b))。 类石墨烯结构层状 $\mathrm{MoS}_{2}$ 场效应晶体管具体应用见 文献[42]。

\section{(b) 集成电路和逻辑器件}

随着电子市场的发展，人们对产品的要求越来 越高, 电子产品也逐渐小型化, 所以研究人员力求 将多种器件集成在同一芯片上。Radisavljevic 等 ${ }^{[43]}$ 首次成功制备了二维半导体 $\mathrm{MoS}_{2}$ 集成电路，该集 成电路可以作为反相器，能够将逻辑 “ 1 ” 变换到逻 辑 “ 0 ”，室温环境下电压增益大于 1 , 故可用于数 字电路中(图 10(a))。Wang 等 ${ }^{[44]}$ 利用制备的双层 $\mathrm{MoS}_{2}$ 晶体管成功制造了集成电路,该晶体管结构表面具 有多种特性, 如电流饱和、高开关比 $\left(>10^{7}\right)$ 、具备导 通状态下的电流密度 $(>23 \mu \mathrm{A} / \mu \mathrm{m})$ (如图 10(b))。

\section{4 检测方面应用}

纳米结构 $\mathrm{MoS}_{2}$ 具有较大的比表面积, 可以有 效吸附气体分子, 所以对气体、光具有较强的敏感 性，可广泛应用于检测方面 ${ }^{[45]}$ 。

Lin 等 ${ }^{[46]}$ 首次发现 $\mathrm{MoS}_{2}$ 纳米片具有固有的过 氧化酶活性，并在过氧化氢作用下可使四甲基联苯 胺(TMB) 呈蓝色, 并且反应取决于温度、 $\mathrm{pH} 、 \mathrm{H}_{2} \mathrm{O}_{2}$ 浓度和反应时间，故通过此种高灵敏度、高选择性 的比色法可以检测血清样品中的葡萄糖(图 11(a))。 Liu 等 ${ }^{[47]}$ 利用硫化法与自组装法相结合成功制备 了 $\mathrm{MoS}_{2}-\mathrm{IO}$ 纳米复合材料, 通过聚乙二醇修饰提 高其稳定性，并成功将二维过渡金属二硫化物复 合材料应用于癌症治疗中(图 11(b))。Wang 等 ${ }^{[48]}$ 在

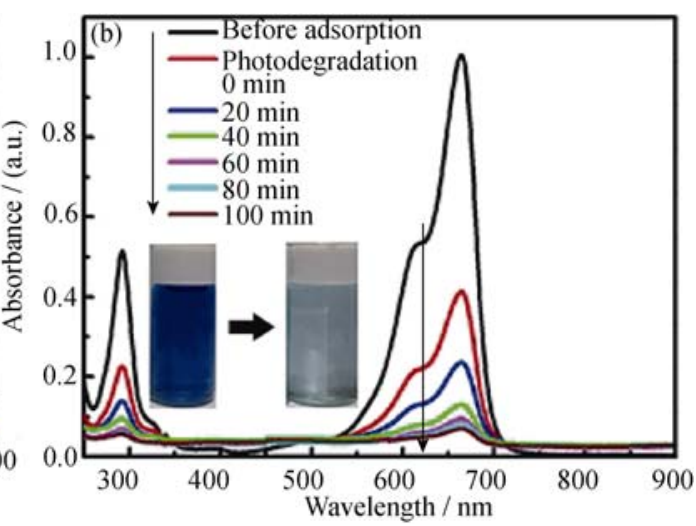

图 8 (a) 甲基蓝水溶液 $\left(\mathrm{MoS}_{2} @ \mathrm{SnO}_{2}\right.$ 纳米花)降解前后的 UV-Vis 吸收图谱 ${ }^{[37]}$ 和(b)甲基蓝水溶液 $\left(\mathrm{MoS}_{2} @ \mathrm{ZnO}\right.$ 纳米异质结) 降解前后的 UV-Vis 吸收图谱 ${ }^{[38]}$

Fig. 8 (a) UV-Vis absorption spectra of a MB solution at room temperature in the presence of $\mathrm{MoS}_{2} @ \mathrm{SnO}_{2}$ nanoflowers ${ }^{[37]}$, (b) UV-Vis absorption spectra of MB solution in the presence of $\mathrm{MoS}_{2} @ \mathrm{ZnO}$ nano-heterojunctions ${ }^{[38]}$ 

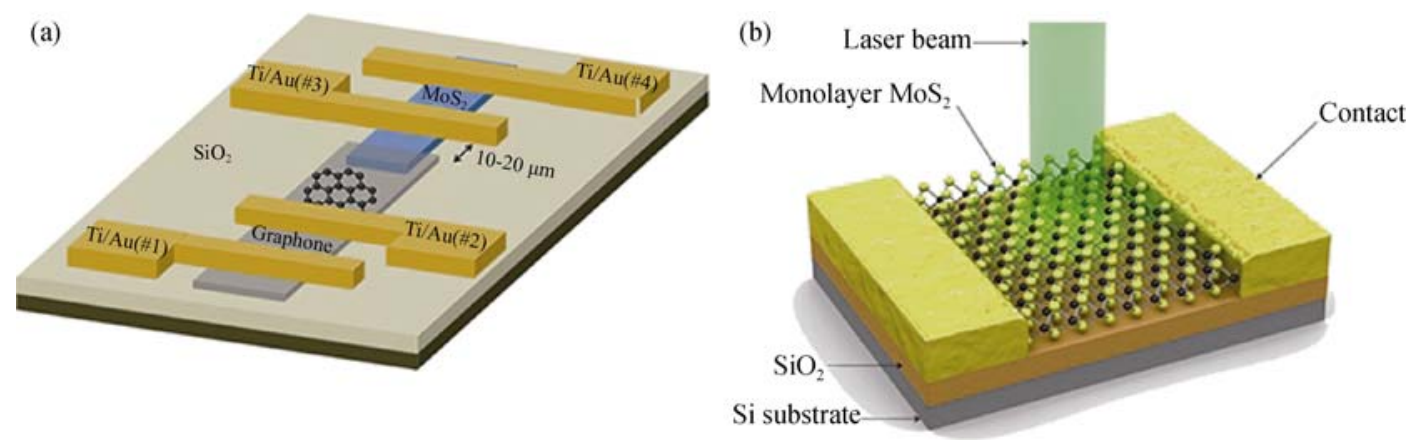

图 9 (a) $\mathrm{MoS}_{2}$ /石墨烯异质结器件的示意图 ${ }^{[40]}$ 和(b) 单层 $\mathrm{MoS}_{2}$ 光电探测器的三维示意图 ${ }^{[41]}$

Fig. 9 (a) Schematic illustration of a $\mathrm{MoS}_{2} /$ graphene heterojunction device ${ }^{[40]}$ and (b) three-dimensional schematic view of the single-layer $\mathrm{MoS}_{2}$ photodetector ${ }^{[41]}$

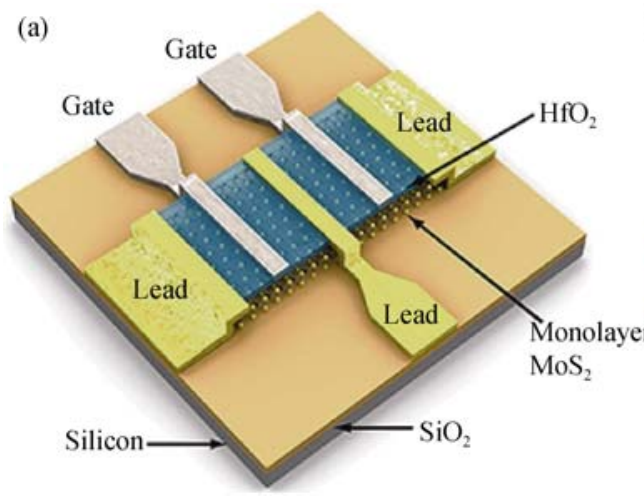

(b)

图 10 (a)单层 $\mathrm{MoS}_{2}$ 的集成电路 ${ }^{[43]}$ 和(b)双层 $\mathrm{MoS}_{2}$ 晶体管的集成电路 ${ }^{[44]}$

Fig. 10 (a) Integrated circuit based on single-layer $\mathrm{MoS}_{2}{ }^{[43]}$ and (b) integrated circuit based on bilayer $\mathrm{MoS}_{2}$ transistors ${ }^{[44]}$

离子溶液中超声、离心混合物成功制备了层状 $\mathrm{MoS}_{2}-$ 硫堇复合材料, 利用 $\mathrm{MoS}_{2}$-硫堇复合材料制备了双 链 DNA 电化学生物传感器, 并应用于人血清的循 环 DNA 检测中，DNA 浓度范围为 $0.09 \sim 1.9 \mathrm{ng} / \mathrm{mL}$ (图 11(c))。

\section{5 锂离子电池方面应用}

$\mathrm{MoS}_{2}$ 材料层间距逐渐增大，不仅降低了层间 的作用力, 且使插入到层间的 $\mathrm{Li}^{+}$的势垒逐渐减弱, 增加电池的充电、放电电容量, 所以纳米结构 $\mathrm{MoS}_{2}$ 可作为锂离子电池的阳极材料 ${ }^{[49]}$ 。由于 $\mathrm{MoS}_{2}$ 是一 种典型的半导体, 导电性能不是很理想, 而碳材料 具有层状结构, 且导电性能良好, 因此现在许多研 究人员将 $\mathrm{MoS}_{2}$ 和 $\mathrm{C}$ 结合起来形成复合材料, 提高 其导电性能，从而提高电学性能。Luo 等 ${ }^{[50]}$ 利用 PVP 作为模板通过水热法成功制备了 $\mathrm{MoS}_{2} / \mathrm{C}$ 微米 球, 结果表明该电极具有良好的电化学性能, 循环 100 次后, 电容量仍为 $575 \mathrm{~mA} / \mathrm{g}$ 。Chang 等 ${ }^{[51]}$ 通过 液相法成功制备了 $\mathrm{MoS}_{2}$ /石墨烯复合物, 结果显示 其具有优异的电学性能。

$\mathrm{Hu}$ 等 ${ }^{[52]}$ 通过水热法与退火相结合成功制备了 三维花状 $\mathrm{MoS}_{2} / \mathrm{C}$ 样品, 结果表明, $\mathrm{MoS}_{2} / \mathrm{C}$ 纳米花
具有优异的电化学性能, 电流浓度为 $100 \mathrm{~mA} / \mathrm{g}$ 时, 电容量约为 $888.1 \mathrm{mAh} / \mathrm{g}$; 电流浓度为 $1000 \mathrm{~mA} / \mathrm{g}$ 时, 电容量仍可达到 $511 \mathrm{mAh} / \mathrm{g}$ (图 12(a))。Wang 等 ${ }^{[53]}$ 通过溶剂热法成功制备了由洋䓤状碳核(OLC)及 $\mathrm{MoS}_{2}$ 纳米花瓣组成的海胆状 $\mathrm{MoS}_{2} / \mathrm{OLC}$ 纳米复合材 料, 并将其作为锂离子电池的阳极材料, 循环 60 次 后, 电流浓度为 $50 \mathrm{~mA} / \mathrm{g}$ 时, 电容量约为 $853 \mathrm{mAh} / \mathrm{g}$ (图 12(b))。

\section{5 结束语}

二硫化钼以其独特的结构性能克服了零带隙 石墨烯材料缺点的同时仍具有其许多优点, 在诸 多领域拥有巨大的潜能，但是新型二硫化钼纳米 材料的研究还有很多科学性问题有待解决。制备方 法方面: 虽然目前已通过多种方法成功制备了 $\mathrm{MoS}_{2}$ 纳米材料, 但是如何改进 $\mathrm{MoS}_{2}$ 纳米材料的制 备方法以达到简单、产率高、重复性好仍然是研究 的重点; 光电器件的应用: 虽然纳米结构二硫化钼 已广泛应用到多种器件中，但是其结构及性能仍 需进一步优化，从而更好的应用于相关领域，逐步 取代其他材料制作器件。 


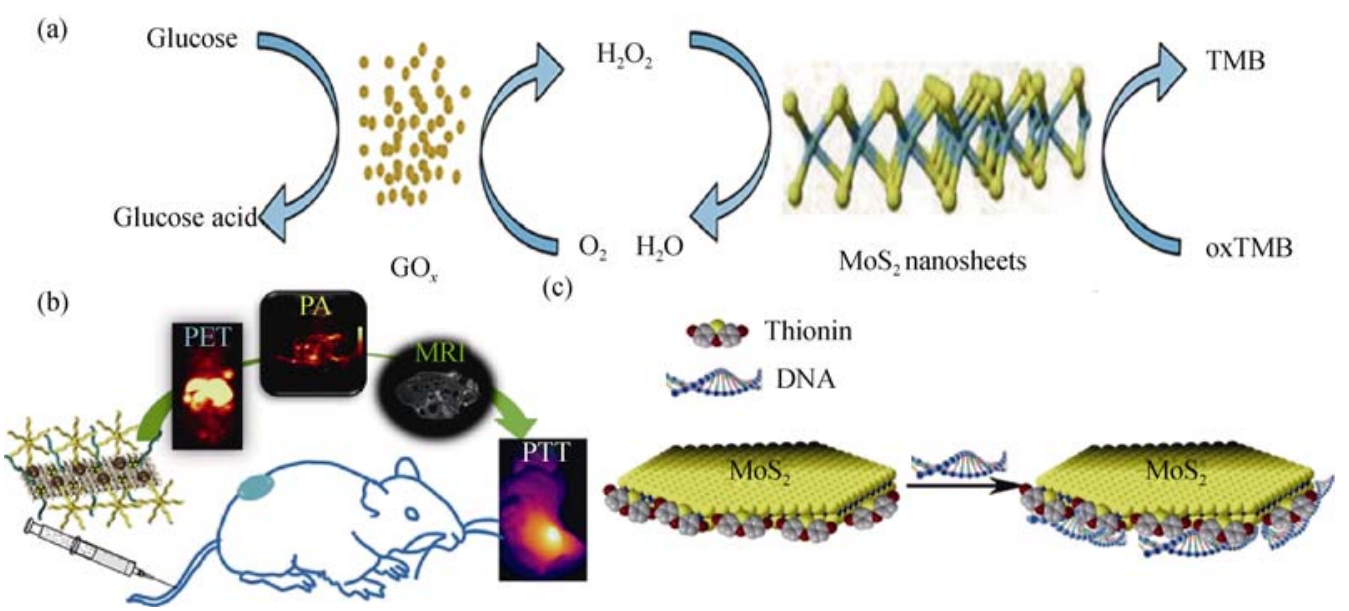

图 11 (a)葡萄糖氧化酶和 $\mathrm{MoS}_{2}$ 纳米片检测葡萄糖的示意图 ${ }^{[46]},(b) \mathrm{MoS}_{2}-\mathrm{IO}-\mathrm{PEG}$ 的活体光照疗法示意图 ${ }^{[47]}$ 和 $(\mathrm{c}) \mathrm{MoS}_{2}$-硫堇电化 学传感器检测 DNA 的示意图 ${ }^{[48]}$

Fig. 11 (a) Schematic illustration of colorimetric detection of glucose by using glucose oxidase $\left(\mathrm{GO}_{x}\right)$ and $\mathrm{MoS}_{2}$ nanosheet ${ }^{[46]}$, (b) schematic illustration of $\mathrm{MoS}_{2}$-IO-PEG in vivo photothermal therapy ${ }^{[47]}$ and (c) schematic illustration of detection of DNA on $\mathrm{MoS}_{2}$-thionin electrochemical sensors ${ }^{[48]}$
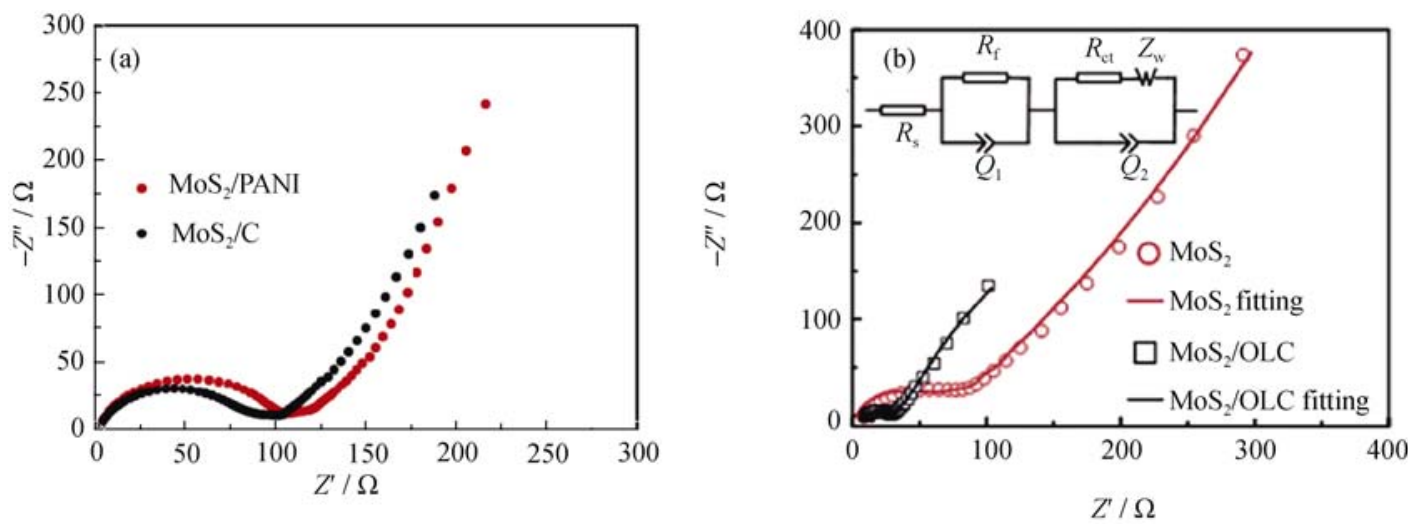

图 12 (a) 三维 $\mathrm{MoS}_{2} / \mathrm{PANI}$ 和 $\mathrm{MoS}_{2} / \mathrm{C}$ 纳米花的阻抗图谱 ${ }^{[52]}$, (b) 商用 $\mathrm{MoS}_{2}$ 和海胆状 $\mathrm{MoS}_{2} / \mathrm{OLC}$ 纳米材料的阻抗图谱 ${ }^{[53]}$

Fig. 12 (a) Nyquist plots of the 3D hierarchical $\mathrm{MoS}_{2} / \mathrm{PANI}$ and $\mathrm{MoS}_{2} / \mathrm{C}$ nanoflowers ${ }^{[52]}$, and (b) nyquist plots of the commercial $\mathrm{MoS}_{2}$ and the synthesized $\mathrm{MoS}_{2} / \mathrm{OLC}$ nano-urchins ${ }^{[53]}$

The inset in (b) is the applied equivalent circuit

\section{参考文献:}

[1] CONLEY H J, WANG B, ZIEGLER J I, et al. Bandgap engineering of strained monolayer and bilayer $\mathrm{MoS}_{2}$. Nano Letters, 2013, 13(8): 3626-3630.

[2] BHATTACHARYYA S, PANDEY T, SINGH A K. Effect of strain on electronic and thermoelectric properties of few layers to bulk $\mathrm{MoS}_{2}$. Nanotechnology, 2014, 25(46): 465701.

[3] MAK K F, LEE C, HONE J, et al. Atomically thin $\mathrm{MoS}_{2}$ : a new direct-gap semiconductor. Physical Review Letters, 2010, 105(13): 13685.

[4] SHI Y M, ZHOU W, LU A Y, et al. Van der Waals epitaxy of $\mathrm{MoS}_{2}$ layers using graphene as growth templates. Nano letters, 2012, 12(6): 2784-2791.

[5] TAN Y H, YU K, YANG T, et al. The combinations of hollow
$\mathrm{MoS}_{2}$ micro@nanospheres: one-step synthesis, excellent photocatalytic and humidity sensing properties. J. Mater. Chem. C, 2014, 2: $5422-5430$.

[6] ZHU H, DU M L, ZHANG M, et al. The design and construction of 3D rose-petal-shaped $\mathrm{MoS}_{2}$ hierarchical nanostructures with structure-sensitive properties. Journal of Materials Chemistry A, 2014, 2(21): 7680-7685.

[7] CHEN Z, CUMMINS D, REINECKE B N, et al. Core-shell $\mathrm{MoO}_{3}-\mathrm{MoS}_{2}$ nanowires for hydrogen evolution: a functional design for electrocatalytic materials. Nano Letters, 2011, 11(10): 4168-4175.

[8] WU Z Z, WANG D Z, LIANG X, et al. Novel hexagonal $\mathrm{MoS}_{2}$ nanoplates formed by solid-state assembly of nanosheets. Journal of Crystal Growth, 2010, 312(12): 1973-1976.

[9] LIN H T, CHEN X Y, Li H L, et al. Hydrothermal synthesis and characterization of $\mathrm{MoS}_{2}$ nanorods. Materials Letters, 2010, 64: 
$1748-1750$.

[10] DEEPAK F L, MAYORAL A, YACAMAN M J. Faceted $\mathrm{MoS}_{2}$ nanotubes and nanoflowers. Materials Chemistry and Physics, 2009, 118(2): 392-397.

[11] YU Y, HUANG S Y, LI Y, et al. Layer-dependent electrocatalysis of $\mathrm{MoS}_{2}$ for hydrogen evolution. Nano Letters, 2014, 14(2): $553-558$.

[12] DING S, ZHANG D, CHEN J S, et al. Facile synthesis of hierarchical $\mathrm{MoS}_{2}$ microspheres composed of few-layered nanosheets and their lithium storage properties. Nanoscale, 2012, 4(1): 95-98.

[13] CAI Y, YANG X, LIANG T, et al. Easy incorporation of singlewalled carbon nanotubes into two-dimensional $\mathrm{MoS}_{2}$ for highperformance hydrogen evolution. Nanotechnology, 2014, 25(46): 465401.

[14] SU S, SUN H F, XU F, et al. Highly sensitive and selective determination of dopamine in the presence of ascorbic acid using gold nanoparticles - decorated $\mathrm{MoS}_{2}$ nanosheets modified electrode. Electroanalysis, 2013, 25(11): 2523-2529.

[15] MANNEBACH E M, DUERLOO K A N, PELLOUCHOUD L A, et al. Ultrafast electronic and structural response of monolayer $\mathrm{MoS}_{2}$ under intense photoexcitation conditions. ACS Nano, 2014, 8(10): 10734-10742.

[16] SHI J P, MA D L, HAN G F, et al. Controllable growth and transfer of monolayer $\mathrm{MoS}_{2}$ on $\mathrm{Au}$ foils and its potential application in hydrogen evolution reaction. ACS Nano, 2014, 8(10): 10196-10204.

[17] ZHAN Y J, LIU Z, NAJMAEI S, et al. Large - area vapor - phase growth and characterization of $\mathrm{MoS}_{2}$ atomic layers on a $\mathrm{SiO}_{2}$ substrate. Small, 2012, 8(7): 966-971.

[18] LIU K K, ZHANG W, LEE Y H, et al. Growth of large-area and highly crystalline $\mathrm{MoS}_{2}$ thin layers on insulating substrates. Nano Letters, 2012, 12(3): 1538-1544.

[19] KONG D, WANG H, CHA J J, et al. Synthesis of $\mathrm{MoS}_{2}$ and $\mathrm{MoSe}_{2}$ films with vertically aligned layers. Nano Letters, 2013, 13(3): 1341-1347.

[20] CAI G M, JIAN J K, CHEN X L, et al. Regular hexagonal $\mathrm{MoS}_{2}$ microflakes grown from $\mathrm{MoO}_{3}$ precursor. Applied Physics A, 2007, 89(3): 783-788

[21] MCDONNELL S, ADDOU R, BUIE C, et al. Defect-dominated doping and contact resistance in $\mathrm{MoS}_{2}$. ACS Nano, 2014, 8(3): 2880-2888.

[22] EDA G, YAMAGUCHI H, VOIRY D, et al. Photoluminescence from chemically exfoliated $\mathrm{MoS}_{2}$. Nano Letters, 2011, 11(12): $5111-5116$

[23] LIU N, KIM P, KIM J H, et al. Large-area atomically thin $\mathrm{MoS}_{2}$ nanosheets prepared using electrochemical exfoliation. ACS Nano, 2014, 8(7): 6902-6910.

[24] YIN Z Y, LI H, LI H, et al. Single-layer $\mathrm{MoS}_{2}$ phototransistors.
ACS Nano, 2011, 6(1): 74-80.

[25] GOPALAKRISHNAN D, DAMIEN D, SHAIJUMON M M. $\mathrm{MoS}_{2}$ quantum dots interspersed exfoliated $\mathrm{MoS}_{2}$ nanosheets. ACS Nano, 2014, 8(5): 5297-5303.

[26] MAIJENBURG A W, REGIS M, HATtORI A N, et al. $\mathrm{MoS}_{2}$ nanocube structures as catalysts for electrochemical $\mathrm{H}_{2}$ evolution from acidic aqueous solutions. ACS Applied Materials \& Interfaces, 2014, 6(3): 2003-2010.

[27] LI Q, WALTER E C, VAM DER VEER W E, et al. Molybdenum disulfide nanowires and nanoribbons by electrochemical/chemical synthesis. The Journal of Physical Chemistry B, 2005, 109(8): 3169-3182.

[28] KIBSGAARD J, CHEN Z B, REINECKE B N, et al. Engineering the surface structure of $\mathrm{MoS}_{2}$ to preferentially expose active edge sites for electrocatalysis. Nature Materials, 2012, 11(11): 963-969.

[29] MA C B, QI X, CHEN B, et al. $\mathrm{MoS}_{2}$ nanoflower-decorated reduced graphene oxide paper for high-performance hydrogen evolution reaction. Nanoscale, 2014, 6(11): 5624-5629.

[30] LIU H, SU X, DUAN C Y, et al. A novel hydrogen peroxide biosensor based on immobilized hemoglobin in 3D flower-like $\mathrm{MoS}_{2}$ microspheres structure. Meterials Letters, 2014, 122: 182-185.

[31] LI G W, LI C S, TANG H, et al. Synthesis and characterization of hollow $\mathrm{MoS}_{2}$ microspheres grown from $\mathrm{MoO}_{3}$ precursors. Journal of Alloys and Compounds, 2010, 501(2): 275-281.

[32] ZHANG L, LOU X W D. Hierarchical $\mathrm{MoS}_{2}$ shells supported on carbon spheres for highly reversible lithium storage. Chemistry-A European Journal, 2014, 20(18): 5219-5223.

[33] LI Y G, WANG H L, XIE L M, et al. $\mathrm{MoS}_{2}$ nanoparticles grown on graphene: an advanced catalyst for the hydrogen evolution reaction. Journal of the American Chemical Society, 2011, 133(19): 7296-7299.

[34] TANG G G, SUN J R, CHEN W, et al. Surfactant-assisted hydrothermal synthesis and tribological properties of flower-like $\mathrm{MoS}_{2}$ nanostructures. Micro \& Nano Letters, 2013, 8(3): 164-168.

[35] RAPOPORT L, FLEISCHER N, TENNE R. Applications of $\mathrm{WS}_{2}$ $\left(\mathrm{MoS}_{2}\right)$ inorganic nanotubes and fullerene-like nanoparticles for solid lubrication and for structural nanocomposites. J. Mater. Chem., 2005, 15(18): 1782-1788.

[36] YUWEN L H, XU F, XUE B, et al. General synthesis of noble metal ( $\mathrm{Au}, \mathrm{Ag}, \mathrm{Pd}, \mathrm{Pt}$ ) nanocrystal modified $\mathrm{MoS}_{2}$ nanosheets and the enhanced catalytic activity of $\mathrm{Pd}-\mathrm{MoS}_{2}$ for methanol oxidation. Nanoscale, 2014, 6: 5762-5769.

[37] LI J Z, YU K, TAN Y H, et al. Facile synthesis of novel $\mathrm{MoS}_{2} @ \mathrm{SnO}_{2}$ hetero-nanoflowers and enhanced photocatalysis and field-emission properties. Dalton Transactions, 2014, 43(34): $13136-13144$

[38] TAN Y H, YU K, LI J Z, et al. $\mathrm{MoS}_{2} @ \mathrm{ZnO}$ nano-heterojunctions 
with enhanced photocatalysis and field emission properties. Journal of Applied Physics, 2014, 116(6): 064305.

[39] YOU X Q, LIU N, LEE C J, et al. An electrochemical route to $\mathrm{MoS}_{2}$ nanosheets for device applications. Materials Letters, 2014, 121: $31-35$.

[40] KWAK J Y, HWANG J, CALDERON B, et al. Electrical characteristics of multilayer $\mathrm{MoS}_{2}$ FET's with $\mathrm{MoS}_{2}$ /graphene heterojunction contacts. Nano Letters, 2014, 14(8): 4511-4516.

[41] LOPEZ-SANCHEZ O, LEMBKE D, KAYCI M, et al. Ultrasensitive photodetectors based on monolayer $\mathrm{MoS}_{2}$. Nature Nanotechnology, 2013, 8(7): 497-501.

[42] LI X, LI J H, WANG X H, et al. Preparation, applications of two-Dimensional graphene-like molybdenum disulfide. Integrated Ferroelectrics, 2014, 158: 26-42.

[43] RADISAVLJEVIC B, WHITWICK M B, KIS A. Integrated circuits and logic operations based on single-layer $\mathrm{MoS}_{2}$. ACS Nano, 2011, 5(12): 9934-9938.

[44] WANG H, YU L, LEE Y H, et al. Integrated circuits based on bilayer $\mathrm{MoS}_{2}$ transistors. Nano Letters, 2012, 12(9): 4674-4680.

[45] LOO A H, BONANNI A, AMBROSI A, et al. Molybdenum disulfide $\left(\mathrm{MoS}_{2}\right)$ nanoflakes as inherently electroactive labels for DNA hybridization detection. Nanoscale, 2014, 6: 11971-11975.

[46] LIN T R, ZHONG L S, GUO L Q, et al. Seeing diabetes: visual detection of glucose based on the intrinsic peroxidase-like activity of $\mathrm{MoS}_{2}$ nanosheets. Nanoscale, 2014, 6(20): 11856-11862.
[47] LIU T, SHI S X, LIANG C, et al. Iron oxide decorated $\mathrm{MoS}_{2}$ nanosheets with double PEGylation for chelator-free radiolabeling and multimodal imaging guided photothermal therapy. ACS Nano, 2015, 9(1): 950-960.

[48] WANG T Y, ZHU R Z, ZHUO J Q, et al. Direct detection of DNA below ppb level based on thionin-functionalized layered $\mathrm{MoS}_{2}$ electrochemical sensors. Analytical Chemistry, 2014, 86(24): 12064-12069.

[49] XU X, FAN Z Y, DING S J, et al. Fabrication of $\mathrm{MoS}_{2}$ nanosheet@ $\mathrm{TiO}_{2}$ nanotube hybrid nanostructures for lithium storage. Nanoscale, 2014, 6(10): 5245-5250.

[50] LUO H, ZHANG L Z, YUE L. Synthesis of $\mathrm{MoS}_{2} / \mathrm{C}$ submicrosphere by PVP-assisted hydrothermal method for lithium ion battery. Advanced Materials Research, 2012, 531: 471-477.

[51] CHANG K, CHEN W X. L-cysteine-assisted synthesis of layered $\mathrm{MoS}_{2}$ /graphene composites with excellent electrochemical performances for lithium ion batteries. ACS Nano, 2011, 5(6): $4720-4728$.

[52] HU L R, REN Y M, YANG H X, et al. Fabrication of 3D hierarchical $\mathrm{MoS}_{2} /$ Polyaniline and $\mathrm{MoS}_{2} / \mathrm{C}$ architectures for lithium-ion battery applications. ACS Applied Materials \& Interfaces, 2014, 6(16): 14644-14652.

[53] WANG Y, XING G Z, HAN Z J, et al. Pre-lithiation of onion-like carbon/ $\mathrm{MoS}_{2}$ nano-urchin anodes for high-performance rechargeable lithium ion batteries. Nanoscale, 2014, 6: 8884-8890. 